\title{
Quadratic Growth Conditions in Optimal Control Problems
}

\author{
J. Frédéric Bonnans and N.P. Osmolovskii \\ This paper is dedicated to Alex Ioffe and Simeon Reich, \\ on the occasion of their 70th and 60th birthdays.
}

\begin{abstract}
This paper provides an analysis of weak, Pontryagin and bounded strong minima satisfying a quadratic growth condition for optimal control problems of ordinary differential equations with constraints on initial-final state and pointwise constraints of two types: (a) inequality and equality mixed (control-state) constraints satisfying the hypothesis of uniform linear independence of gradients with respect to control $u$ of active constraints and (b) inequality control constraints satisfying the hypothesis of uniform positive linear independence of gradients of active constraints.
\end{abstract}

\section{Mixed control-state constraints with linearly independent gradients with respect to control}

In this section we recall results due to N.P. Osmolovskii. We discuss the quadratic conditions $[\mathbf{1 1}$, Sec. S.2], [16] of a minimum for an optimal control problem with mixed (control-state) equality and inequality type constraints, satisfying the hypothesis of linear independence of gradients with respect to control $u$ of active constraints. The necessary condition for a minimum is that the maximum of the quadratic forms on the set of critical variations is non-negative, where the maximum is taken over the set of multipliers satisfying Pontryagin's principle (by contrast to abstract optimization problems where the maximum is on the larger set of Lagrange multipliers, see $[\mathbf{9}, \mathbf{1 1}])$. An appropriate strengthening of this condition turns out to be sufficient for a strong minimum, provided that the admissible controls are bounded by any large constant. We call bounded strong the minimum of this type. The sufficient conditions of bounded strong minimum guarantee the so-called "bounded strong quadratic growth".

1.1. Pontryagin and bounded strong minima. First order necessary conditions. Let $\left[t_{0}, t_{f}\right]$ denote a fixed time interval. Let $\mathcal{U}:=L^{\infty}\left(t_{0}, t_{f} ; \mathbb{R}^{m}\right)$ and

2000 Mathematics Subject Classification. Primary 49K15; Secondary 90C48.

This work started during a visit of the second author at INRIA-Saclay in the year 2007, thanks to an INRIA grant.

The second author was supported by grants RFBR 8-01-00685 and NSh-3233.2008.1.

(C)2010 J. F. Bonnans, N. P. Osmolovskii

85 
$\mathcal{Y}:=W^{1, \infty}\left(t_{0}, t_{f} ; \mathbb{R}^{n}\right)$ denote the control and state space. Set $\mathcal{W}:=\mathcal{U} \times \mathcal{Y}$. When needed we denote $w=(u, y)$ the elements of $\mathcal{W}$. Define the norm of element $w \in \mathcal{W}$ by $\|w\|_{\mathcal{W}}=\|u\|_{\infty}+\|y\|_{1, \infty}=\operatorname{supess}_{\left[t_{0}, t_{f}\right]}|u(t)|+|y(0)|+\operatorname{supess}_{\left[t_{0}, t_{f}\right]}|\dot{y}(t)|$. Consider the following optimal control problem:

$$
\begin{aligned}
& J(w):=\Phi_{0}\left(y\left(t_{0}\right), y\left(t_{f}\right)\right) \rightarrow \min , \\
& \Phi_{i}\left(y\left(t_{0}\right), y\left(t_{f}\right)\right) \leq 0, i=1, \ldots, r_{1}, \\
& \Phi_{i}\left(y\left(t_{0}\right), y\left(t_{f}\right)\right)=0, i=r_{1}+1, \ldots, r, \\
& \dot{y}(t)=f(t, u(t), y(t)) \text { for a.a. } t \in\left(t_{0}, t_{f}\right), \\
& g_{i}(t, u(t), y(t)) \leq 0, \text { for a.a. } t \in\left(t_{0}, t_{f}\right), i=1, \ldots, q_{1}, \\
& g_{i}(t, u(t), y(t))=0, \text { for a.a. } t \in\left(t_{0}, t_{f}\right), i=q_{1}+1, \ldots, q,
\end{aligned}
$$

where $r=r_{1}+r_{2}, q=q_{1}+q_{2}, r_{1}, r_{2}, q_{1}$, and $q_{2}$ are nonnegative integers. We assume that $f: \mathbb{R} \times \mathbb{R}^{m} \times \mathbb{R}^{n} \rightarrow \mathbb{R}^{n}, g_{i}: \mathbb{R} \times \mathbb{R}^{m} \times \mathbb{R}^{n} \rightarrow \mathbb{R}, i=1, \ldots q$, and $\Phi_{i}: \mathbb{R}^{n} \times \mathbb{R}^{n} \rightarrow \mathbb{R}, i=0, \ldots, r$ are twice continuously differentiable $\left(C^{2}\right)$ mappings. We also assume that the mixed constraints (1.5) and (1.6) satisfy the following qualification condition: for any point $(t, u, y) \in \mathbb{R} \times \mathbb{R}^{m} \times \mathbb{R}^{n}$ such that

$$
g_{i}(t, u, y) \leq 0, i=1, \ldots, q_{1}, \quad g_{i}(t, u, y)=0, i=q_{1}+1, \ldots, q,
$$

the gradients $g_{i u}(t, u, y), i \in I_{g}(t, u, y)$ are linearly independent, where

$$
I_{g}(t, u, y)=\left\{i \in\{1, \ldots, q\} \mid g_{i}(t, u, y)=0\right\} .
$$

We refer to problem (1.1)-(1.6) as problem $(\mathrm{P})$. Elements of $\mathcal{W}$ satisfying (1.2)(1.6) are said to be feasible. The set of feasible points is denoted by $F(P)$.

Let us recall two concepts of minimum. We say that $\bar{w}=(\bar{u}, \bar{y}) \in F(P)$ is a bounded strong minimum (see [11, Sec. S2] and [14, p. 291]) if $J(\bar{w}) \leq J\left(w^{k}\right)$ for large enough $k$ for any sequence $w^{k} \in F(P)$, bounded in $\mathcal{W}$, such that $y^{k} \rightarrow \bar{y}$ uniformly. We say that $\bar{w} \in F(P)$ is a Pontryagin minimum (see [11, p. 156] and [14, p. 285]) if $J(\bar{w}) \leq J\left(w^{k}\right)$ for large enough $k$ for any sequence $w^{k} \in F(P)$, bounded in $\mathcal{W}$, such that $y^{k} \rightarrow \bar{y}$ uniformly and $\left\|u^{k}-\bar{u}\right\|_{1} \rightarrow 0$, where $\|u\|_{1}=\int_{t_{0}}^{t_{f}}|u(t)| \mathrm{d} t$.

Equivalently, $\bar{w}$ is a bounded strong minimum iff for any $N>0$, there exists $\varepsilon>0$ such that if $w \in F(P)$ is such that $\|u\|_{\infty} \leq N,\|y-\bar{y}\|_{\infty} \leq \varepsilon$, we have that $J(\bar{w}) \leq J(w)$. A point $\bar{w}$ is a Pontryagin minimum iff for any $N>0$, there exists $\varepsilon>0$ such that if $w \in F(P)$ is such that $\|u\|_{\infty} \leq N,\|y-\bar{y}\|_{\infty} \leq \varepsilon$, and $\|u-\bar{u}\|_{1}<\varepsilon$, we have that $J(\bar{w}) \leq J(w)$. Finally, recall that a weak minimum is a local minimum in $\mathcal{W}$. Obviously, a bounded strong minimum is a Pontryagin minimum, and the latter is a weak minimum.

Let us formulate a first order necessary condition of a weak minimum. We denote by $\mathbb{R}^{n *}$ the dual of $\mathbb{R}^{n}$ (identified with the set of $n$-dimensional horizontal vectors). Define the endpoint Lagrange function

$$
\Phi^{\mu}\left(y_{0}, y_{f}\right)=\sum_{i=0}^{r} \mu_{i} \Phi_{i}\left(y_{0}, y_{f}\right)
$$

where $\mu=\left(\mu_{0}, \ldots, \mu_{r}\right) \in \mathbb{R}^{(r+1) *}, y\left(t_{0}\right)=y_{0}$, and $y\left(t_{f}\right)=y_{f}$. In the sequel, we shall use the abbreviation $\left(y_{0}, y_{f}\right)=\eta$. Consider the Hamiltonian function $H: \mathbb{R} \times \mathbb{R}^{m} \times \mathbb{R}^{n} \times \mathbb{R}^{n *} \rightarrow \mathbb{R}$ and the augmented Hamiltonian function $H^{a}$ : $\mathbb{R} \times \mathbb{R}^{m} \times \mathbb{R}^{n} \times \mathbb{R}^{n *} \times \mathbb{R}^{q *} \rightarrow \mathbb{R}$ defined by

$$
H(t, u, y, p)=p f(t, u, y), \quad H^{a}(t, u, y, p, \lambda)=H(t, u, y, p)+\lambda g(t, u, y),
$$


where $g=\left(g_{1}, \ldots, g_{q}\right)^{*} \in \mathbb{R}^{q}, \lambda=\left(\lambda_{1}, \ldots, \lambda_{q}\right) \in \mathbb{R}^{q *}$. For $\bar{w}=(\bar{u}, \bar{y}) \in F(P)$, denote by $\Lambda(\bar{w})$ the set of all tuples of Lagrange multipliers

$$
\nu=(p, \lambda, \mu) \in W^{1, \infty}\left(t_{0}, t_{f} ; \mathbb{R}^{n *}\right) \times L^{\infty}\left(t_{0}, t_{f} ; \mathbb{R}^{q *}\right) \times \mathbb{R}^{(r+1) *},
$$

normalized by condition $|\mu|=1$, such that the following relations hold:

$$
\begin{aligned}
& \mu_{i} \geq 0, i=0, \ldots, r_{1}, \mu_{i} \Phi_{i}(\bar{\eta})=0, i=1, \ldots, r_{1}, \\
& \lambda_{i}(t) \geq 0, \lambda_{i}(t) g_{i}(t, \bar{w}(t))=0, \text { for a.a. } t \in\left(t_{0}, t_{f}\right), i=1, \ldots, q_{1}, \\
& -\dot{p}(t)=H_{y}^{a}(t, \bar{w}(t), p(t), \lambda(t)), \text { for a.a. } t \in\left(t_{0}, t_{f}\right), \\
& p\left(t_{0}\right)=-\Phi_{y_{0}}^{\mu}(\bar{\eta}), p\left(t_{f}\right)=\Phi_{y_{f}}^{\mu}(\bar{\eta}), \\
& H_{u}^{a}(t, \bar{w}(t), p(t), \lambda(t))=0, \text { for a.a. } t \in\left(t_{0}, t_{f}\right),
\end{aligned}
$$

where $\bar{\eta}=\left(\bar{y}\left(t_{0}\right), \bar{y}\left(t_{f}\right)\right)$. The following result is well-known, see $[\mathbf{5}, \mathbf{1 3}],[\mathbf{1 4}$, pp. 148-151], [16, 10], [6, Ch. 3, Thm. 4.2], [7]:

THEOREM 1.1. If $\bar{w}$ is a weak minimum, then $\Lambda(\bar{w})$ is a nonempty finitedimensional compact set, and the projector $(p, \lambda, \mu) \rightarrow \mu$ is injective on $\Lambda(\bar{w})$.

Let us recall the formulation of Pontryagin's principle at the point $\bar{w} \in F(P)$.

Definition 1.2. We say that $\bar{w}=(\bar{u}, \bar{y}) \in F(P)$ satisfies Pontryagin's principle if there exists $\nu=(p, \lambda, \mu)$ with $\mu \neq 0$, satisfying relations (1.10)-(1.15) and such that for a.a. $t \in\left(t_{0}, t_{f}\right)$ the following inequality holds:

$$
H(t, \bar{u}(t), \bar{y}(t), p(t)) \leq H(t, v, \bar{y}(t), p(t)), \text { for all } v \in U(t, y(t)), \text { a.a. } t \in\left(t_{0}, t_{f}\right)
$$

where $U(t, y)$ is the set of $u \in \mathbb{R}^{m}$ such that the point $(t, u, y)$ satisfies mixed constraints (1.7).

Note that (1.10)-(1.14) and (1.16) imply (1.15). The following theorem holds, $[\mathbf{1 1}],[13],[14],[16]$.

Theorem 1.3. A Pontryagin minimum satisfies Pontryagin's principle.

Denote by $M(\bar{w})$ the set of all $\lambda=(p, \lambda, \mu) \in \Lambda(\bar{w})$ satisfying inequality (1.16) of Pontryagin's principle. Obviously, $M(\bar{w}) \subset \Lambda(\bar{w})$, and the condition $M(\bar{w}) \neq \emptyset$ is equivalent to Pontryagin's principle.

1.2. Second order necessary conditions of Pontryagin minimum. By $\delta w=(\delta u, \delta y)$ we denote a variation, i.e., an arbitrary element of the space $\mathcal{W}$. A variation (direction) $\delta w=(\delta u, \delta y) \in \mathcal{W}$ is said to be critical, [5], at the point $\bar{w} \in F(P)$ if the following relations hold

$$
\begin{aligned}
& \Phi_{i}^{\prime}(\bar{\eta}) \delta \eta \leq 0, i \in I_{\Phi}(\bar{\eta}) \cup\{0\} ; \Phi_{i}^{\prime}(\bar{\eta}) \delta \eta=0, i=r_{1}+1, \ldots, r, \\
& \delta \dot{y}(t)=f_{w}(t, \bar{w}(t)) \delta w(t) \text {, for a.a. } t \in\left(t_{0}, t_{f}\right) \text {, } \\
& g_{i w}(t, \bar{w}(t)) \delta w(t) \chi_{\left\{g_{i}(t, \bar{w})=0\right\}} \leq 0 \text {, for a.a. } t \in\left(t_{0}, t_{f}\right), i=1, \ldots, q_{1} \text {, } \\
& g_{i w}(t, \bar{w}(t)) \delta w(t)=0 \text {, for a.a. } t \in\left(t_{0}, t_{f}\right), i=q_{1}+1, \ldots, q \text {, }
\end{aligned}
$$

where $I_{\Phi}(\bar{\eta})=\left\{i \in\left\{1, \ldots, r_{1}\right\} \mid \Phi_{i}(\bar{\eta})=0\right\}$ is the set of active indices, $\chi_{\left\{g_{i}(t, \bar{w})=0\right\}}$ is the characteristic function of the set $\left\{t \in\left[t_{0}, t_{f}\right] \mid g_{i}(t, \bar{w}(t))=0\right\}, i=1, \ldots, q_{1}$, and $\delta \eta=\left(\delta y\left(t_{0}\right), \delta y\left(t_{f}\right)\right)$. Denote by $\mathcal{C}(\bar{w})$ the set of all critical directions $\delta w \in \mathcal{W}$ at the point $\bar{w}$. Obviously, $\mathcal{C}(\bar{w})$ is a convex cone in $\mathcal{W}$. We call it the critical cone. 
For any $\nu=(p, \lambda, \mu) \in \Lambda(\bar{w})$, let us define a quadratic form at the point $\bar{w}$ by the relation

$$
\begin{aligned}
\Omega(\bar{w}, \nu ; \delta w)= & \frac{1}{2}\left\langle\left(\Phi^{\mu}\right)^{\prime \prime}(\bar{\eta}) \delta \eta, \delta \eta\right\rangle \\
& +\frac{1}{2} \int_{t_{0}}^{t_{1}}\left\langle H_{w w}^{a}(t, \bar{w}(t), p(t), \lambda(t)) \delta w(t), \delta w(t)\right\rangle \mathrm{d} t .
\end{aligned}
$$

The following Theorem holds, see [11, Sec. S.2] (proofs are given in [16]):

Theorem 1.4. If $\bar{w} \in F(P)$ is a Pontryagin minimum, then the set $M(\bar{w})$ is nonempty and

$$
\max _{\nu \in M(\bar{w})} \Omega(\bar{w}, \nu ; \delta w) \geq 0 \quad \text { for all } \quad \delta w \in \mathcal{C}(\bar{w}) .
$$

1.3. Bounded strong $\gamma$-sufficiency. Second order sufficient conditions of bounded strong minimum. In order to define a bounded strong $\gamma$-sufficiency, we must define the so-called 'order function'. A function $\Gamma: \mathbb{R}^{m} \rightarrow \mathbb{R}$ is said to be an order function if there exists a number $\varepsilon_{\Gamma}>0$ such that (a) $\Gamma(v)=\frac{1}{2}|v|^{2}$ if $|v|<\varepsilon_{\Gamma}$; (b) $\Gamma(v)>0$ if $|v| \geq \varepsilon_{\Gamma}$; (c) $\Gamma(v)$ is Lipschitz continuous on each compact set $C \subset \mathbb{R}^{m}$. Obviously, the function $\Gamma(v)=\frac{1}{2}|v|^{2}$ is an order function. For an arbitrary order function $\Gamma(v)$, we set

$$
\gamma(\delta w)=\|\delta y\|_{\infty}^{2}+\int_{t_{0}}^{t_{f}} \Gamma(\delta u(t)) \mathrm{d} t .
$$

Then the functional $\gamma: \mathcal{W} \rightarrow \mathbb{R}$ is called the higher order $[\mathbf{1 1}]$.

Let us fix a pair $\bar{w}=(\bar{u}, \bar{y}) \in F(P)$. For any $\delta w=(\delta u, \delta y) \in \mathcal{W}$ we set $\delta f=f(t, \bar{w}(t)+\delta w(t))-f(t, \bar{w}(t))$, i.e., $\delta f$ is the increment of the function $f$ at the point $\bar{w}(t)$ which corresponds to the variation $\delta w(t)$. Similarly, we set $\delta \Phi_{0}=$ $\Phi_{0}(\bar{\eta}+\delta \eta)-\Phi_{0}(\bar{\eta})$, etc. Let us define the violation function $[\mathbf{1 1}]$

$$
\sigma(\delta w)=(\delta J)_{+}+\sum_{i=1}^{r_{1}} \Phi_{i}(\bar{\eta}+\delta \eta)_{+}+\sum_{i=r_{1}+1}^{r}\left|\Phi_{i}(\bar{\eta}+\delta \eta)\right|+\|\delta \dot{y}-\delta f\|_{1},
$$

where $\delta \eta=\left(\delta y_{0}, \delta y_{f}\right)=\left(\delta y\left(t_{0}\right), \delta y\left(t_{f}\right)\right),(\delta J)_{+}=\left(\Phi_{0}(\bar{\eta}+\delta \eta)-\Phi_{0}(\bar{\eta})\right)_{+}, \alpha_{+}=$ $\max \{\alpha, 0\}$.

We say that $\left\{\delta w_{k}\right\}$ is a bounded strong sequence of variations if $\sup _{k}\left\|\delta u_{k}\right\|_{\infty}<$ $\infty,\left\|\delta y_{k}\right\|_{\infty} \rightarrow 0(k \rightarrow \infty)$. Denote by $S$ the set of all bounded strong sequences of variations satisfying the relations

$$
\begin{aligned}
& g_{i}\left(t, \bar{w}(t)+\delta w_{k}(t)\right) \leq 0, \text { for a.a. } t \in\left(t_{0}, t_{f}\right), i=1, \ldots, q_{1}, \\
& g_{i}\left(t, \bar{w}(t)+\delta w_{k}(t)\right)=0, \text { for a.a. } t \in\left(t_{0}, t_{f}\right), i=q_{1}+1, \ldots, q,
\end{aligned}
$$

for all $k$. We say, $[\mathbf{1 1}]$, that a bounded strong $\gamma$-sufficiency holds at the point $\bar{w}$ if there exists $\alpha>0$ such that for any sequence $\left\{\delta w_{k}\right\} \in S$ we have $\sigma\left(\delta w_{k}\right) \geq \alpha \gamma\left(\delta w_{k}\right)$ for all sufficiently large $k$.

Equivalently, a bounded strong $\gamma$-sufficiency holds iff there exist $\alpha>0$ such that for any $N>0$ there exists $\varepsilon>0$ such that if $w \in \mathcal{W}$ satisfies the conditions (1.5), (1.6) and $\|y-\bar{y}\|_{\infty}<\varepsilon,\|u\|_{\infty}<N$, we have that $\sigma(w-\bar{w}) \geq \alpha \gamma(w-\bar{w})$. Obviously, a bounded strong $\gamma$-sufficiency implies a strict bounded strong minimum.

Let $\nu=(p, \lambda, \mu) \in M(\bar{w})$. We say that the function $H(t, v, \bar{y}(t), p(t))$ satisfies a growth condition of the order $\Gamma$ if there exists $\alpha>0$ such that we have

$$
\left\{\begin{array}{l}
H(t, v, \bar{y}(t), p(t))-H(t, \bar{u}(t), \bar{y}(t), p(t)) \geq \alpha \Gamma(v-\bar{u}(t)) \\
\text { for all } v \in U(t, \bar{y}(t)), \text { a.a. } t \in\left[t_{0}, t_{f}\right] .
\end{array}\right.
$$


For any $\alpha>0$, denote by $M_{\alpha \Gamma}(\bar{w})$ the set of all $\nu=(p, \lambda, \mu) \in M(\bar{w})$ satisfying condition (1.23). The following Theorem holds, see [11, Sec. S.2] (proofs are given in $[\mathbf{1 6}])$ :

TheOREM 1.5. Let $\Gamma$ be an order function, and $\gamma$ be the corresponding higher order. Then the bounded strong $\gamma$-sufficiency at the point $\bar{w}=(\bar{u}, \bar{y}) \in F(P)$ is equivalent to the following condition: there exists $\alpha>0$ such that the set $M_{\alpha \Gamma}(\bar{w})$ is nonempty and

$$
\max _{\nu \in M_{\alpha \Gamma}(\bar{w})} \Omega(\bar{w}, \nu ; \delta w) \geq \alpha\left(\left|\delta y_{0}\right|^{2}+\|\delta u\|_{2}^{2}\right) \quad \text { for all } \quad \delta w \in \mathcal{C}(\bar{w}),
$$

where $\delta y_{0}=\delta y\left(t_{0}\right)$ and $\|\delta u\|_{2}=\left(\int_{t_{0}}^{t_{f}}\langle\delta u(t), \delta u(t)\rangle \mathrm{d} t\right)^{1 / 2}$.

1.4. Second order conditions of a weak minimum. Weak quadratic sufficiency. The results of this section are contained in $[\mathbf{1 6}]$.

THeOREM 1.6. If $\bar{w} \in F(P)$ is a weak minimum, then the set $\Lambda(\bar{w})$ is nonempty and

$$
\max _{\nu \in \Lambda(\bar{w})} \Omega(\bar{w}, \nu ; \delta w) \geq 0 \quad \text { for all } \quad \delta w \in \mathcal{C}(\bar{w}) .
$$

Assume that $\bar{w} \in F(P)$ is such that $\Lambda(\bar{w})$ is nonempty. Let $\nu=(p, \lambda, \mu) \in \Lambda(\bar{w})$. We say that the function $H(t, v, \bar{y}(t), p(t))$ satisfies a local quadratic growth condition if there exist $\alpha>0$ and $\varepsilon>0$ such that for a.a. $t \in\left[t_{0}, t_{f}\right]$ we have

$$
\left\{\begin{array}{l}
H(t, v, \bar{y}(t), p(t))-H(t, \bar{u}(t), \bar{y}(t), p(t)) \geq \frac{1}{2} \alpha|v-\bar{u}(t)|^{2} \\
\text { for all } v \in U(t, \bar{y}(t)), \text { such that }|v-\bar{u}(t)|<\varepsilon
\end{array}\right.
$$

An interesting and nontrivial question is to find a characterization of this condition in terms of Legendre type conditions.

For any $\alpha>0$ denote by $\Lambda(\bar{w}, \alpha)$ the set of all $\nu=(p, \lambda, \mu) \in \Lambda(\bar{w})$ such that the condition (1.26) is satisfied with $\varepsilon=\alpha$ for a.a. $t \in\left[t_{0}, t_{f}\right]$.

We say, [11], that a weak quadratic sufficiency holds at the point $\bar{w} \in F(P)$ if there exists $\alpha>0$ such that for any sequence $\left\{\delta w_{k}\right\}$ in $\mathcal{W}$ satisfying (1.22) and such that $\left\|\delta w_{k}\right\|_{\mathcal{W}} \rightarrow 0(k \rightarrow \infty)$, we have: $\sigma\left(\delta w_{k}\right) \geq \alpha\left\|\delta w_{k}\right\|_{2}^{2}$ for all sufficiently large $k$.

Equivalently, a weak quadratic sufficiency holds at the point $\bar{w}$ iff there exist $\alpha>0$ and $\varepsilon>0$ such that for any $w \in \mathcal{W}$ satisfying (1.5), (1.6) and the condition $\|w-\bar{w}\|_{\mathcal{W}}<\varepsilon$, we have that $\sigma(w-\bar{w}) \geq \alpha\|w-\bar{w}\|_{2}^{2}$. Obviously, a weak quadratic sufficiency implies a strict weak minimum.

THEOREM 1.7. A weak quadratic sufficiency at the point $\bar{w} \in F(P)$ is equivalent to the following condition: there exists $\alpha>0$ such that the set $\Lambda(\bar{w}, \alpha)$ is nonempty and

$$
\max _{\nu \in \Lambda(\bar{w}, \alpha)} \Omega(\bar{w}, \nu ; \delta w) \geq \alpha\left(\left|\delta y_{0}\right|^{2}+\|\delta u\|_{2}^{2}\right) \quad \text { for all } \quad \delta w \in \mathcal{C}(\bar{w}) .
$$

REMARK 1.8. All results of this section hold true if, in problem (1.1)-(1.6), the cost function (1.1) is replaced by the function of the form $J(w)=\Phi_{0}\left(y\left(t_{0}\right), y\left(t_{f}\right)\right)+$ $\int_{t_{0}}^{t_{f}} F(t, u(t), y(t)) \mathrm{d} t$ (where $F: \mathbb{R} \times \mathbb{R}^{m} \times \mathbb{R}^{n} \rightarrow \mathbb{R}$ is $C^{2}$ mapping) with corresponding changes in definitions of the functions $H, H^{a}, \Omega, \sigma$ and the sets $\Lambda(\bar{w}), M(\bar{w})$, and $\mathcal{C}(\bar{w})$. A similar statement is true for any of initial-final state constraints (1.2). 
REMARK 1.9. Set $\mathcal{W}_{2}=\mathcal{U}_{2} \times \mathcal{Y}_{2}$, where $\mathcal{U}_{2}:=L^{2}\left(t_{0}, t_{f} ; \mathbb{R}^{m}\right)$ is the space of square integrable functions $u:\left[t_{0}, t_{f}\right] \rightarrow \mathbb{R}^{m}$ and $\mathcal{Y}_{2}:=W^{1,2}\left(t_{0}, t_{f} ; \mathbb{R}^{n}\right)$ is the space of absolutely continuous functions $y:\left[t_{0}, t_{f}\right] \rightarrow \mathbb{R}^{n}$ with square integrable first derivative. For a feasible point $\bar{w}$, denote by $\mathcal{C}_{2}(\bar{w})$ the set of all $\delta w \in \mathcal{W}$ satisfying relations $(1.17)$. Obviously, $\mathcal{C}_{2}(\bar{w})$ is a convex cone in $\mathcal{W}_{2}$, and $\mathcal{C}(\bar{w}) \subset \mathcal{C}_{2}(\bar{w})$. One may show that condition (1.19) is equivalent to

$$
\max _{\nu \in M(\bar{w})} \Omega(\bar{w}, \nu ; \delta w) \geq 0 \quad \text { for all } \quad \delta w \in \mathcal{C}_{2}(\bar{w}),
$$

and therefore, in view of Theorem 1.4, conditions $M(\bar{w}) \neq \emptyset$ and (1.28) are also necessary conditions of Pontryagin minimum at the point $\bar{w}$.

Recall that vectors $a^{1}, \ldots, a^{k}$ in $\mathbb{R}^{n}$ are said to be positively linearly independent if the relations $\sum_{i=1}^{k} \alpha^{i} a^{i}=0, \alpha_{i} \geq 0, i=1, \ldots, k$ imply $\alpha_{i}=0, i=1, \ldots, k$. There is an open question: do the results of this section hold if instead of condition of linear independence of gradients $g_{i u}(t, u, y), i \in I(t, u, y)$ at each point $(t, u, y) \in \mathbb{R}^{m+n+1}$ satisfying relations (1.7), we assume positive linear independence of these gradients at the same points? Below we will give a partial answer to this question. First, we will analyze the relations between bounded strong and Pontryagin minima in optimal control problems with initial final state constraints and control constraints of the form $u(t) \in U$, where $U$ is an arbitrary closed set in $\mathbb{R}^{m}$.

\section{Control constraint of the form $u(t) \in U$ and initial-final state constraints}

In the remainder of this paper we present results which will be published in $[\mathbf{1}]$.

2.1. Pontryagin's principle. Let $[0, T]$ be a fixed time interval, and let $\mathcal{U}:=$ $L^{\infty}\left(0, T ; \mathbb{R}^{m}\right)$ and $\mathcal{Y}:=W^{1, \infty}\left(0, T ; \mathbb{R}^{n}\right)$ denote the control and state space. Set $\mathcal{W}:=\mathcal{U} \times \mathcal{Y}$. Again, we denote $w=(u, y), \bar{w}=(\bar{u}, \bar{y})$, etc. the elements of $\mathcal{W}$. Similarly we denote when needed $\eta=(y(0), y(T)), \bar{\eta}=(\bar{y}(0), \bar{y}(T))$, etc. The cost function is defined by

$$
J(w):=\int_{0}^{T} \ell(u(t), y(t)) \mathrm{d} t+\phi(\eta),
$$

where $\ell: \mathbb{R}^{m} \times \mathbb{R}^{n} \rightarrow \mathbb{R}$ (running cost) and $\phi: \mathbb{R}^{n} \times \mathbb{R}^{n} \rightarrow \mathbb{R}$ (initial-final cost) are twice continuously differentiable $\left(C^{2}\right)$ mappings. Consider the state equation

$$
\dot{y}(t)=f(u(t), y(t)) \text { for a.a. } t \in[0, T] ;
$$

where $f: \mathbb{R}^{m} \times \mathbb{R}^{n} \rightarrow \mathbb{R}^{n}$ is a Lipschitz and $C^{2}$ mapping. We know that the state equation (2.2) has for any $u \in \mathcal{U}$ and given initial condition $y(0)=y_{0}$ a unique solution denoted $y_{u, y_{0}} \in \mathcal{Y}$.

We consider problems having both control constraints

$$
u(t) \in U, \quad \text { for a.a. } t \in(0, T),
$$

where $U$ is a closed subset of $\mathbb{R}^{m}$, and initial-final state constraints of the form

$$
\Phi(\eta) \in K
$$

where $\Phi: \mathbb{R}^{2 n} \rightarrow \mathbb{R}^{r}, r=r_{1}+r_{2}, r_{1}$ and $r_{2}$ are nonnegative integers, and $K=$ $\{0\}_{\mathbb{R}^{r_{1}}} \times \mathbb{R}_{-}^{r_{2}}$. In other words, there is a finite number of equality and inequality constraints on the initial-final state:

$$
\Phi_{i}(\eta)=0, \quad i=1, \ldots, r_{1}, \quad \Phi_{i}(\eta) \leq 0, \quad i=r_{1}+1, \ldots, r .
$$


Consider the following optimal control problem:

$$
\operatorname{Min}_{w \in \mathcal{W}} J(w) \text { subject to }(2.2)-(2.4) \text {. }
$$

Elements of $\mathcal{W}$ satisfying (2.2)-(2.4) are said to be feasible. The set of feasible points is denoted by $F(P)$.

We say that a (bounded strong, Pontryagin, weak) minimum (see definitions in section 1.1) $\bar{w}$ satisfies the quadratic growth condition if there exists $\alpha>0$ (depending on the size $N$ of the $L^{\infty}$ neighborhood for the control in the case of a bounded strong or Pontryagin minimum) such that $\bar{w}$ is a minimum of the same kind for the cost function

$$
J_{\alpha}(u, y):=\int_{0}^{T} \ell_{\alpha}(u(t), y(t)) \mathrm{d} t+\phi(\eta)
$$

where

$$
\ell_{\alpha}(t, u, y):=\ell(u, y)-\frac{1}{2} \alpha\left[|u-\bar{u}(t)|^{2}+|y-\bar{y}(t)|^{2}\right] .
$$

Then we say that the (bounded strong, Pontryagin, weak) quadratic growth condition is satisfied. So for instance the quadratic growth condition for a weak minimum $\bar{w}$ (we speak then of weak quadratic growth) means that

$$
\left\{\begin{array}{l}
\text { There exist } \alpha>0, \varepsilon>0: J(w) \geq J(\bar{w})+\frac{1}{2} \alpha\|w-\bar{w}\|_{2}^{2}, \\
\text { for all } w \in F(P),\|w-\bar{w}\|_{\infty}<\varepsilon
\end{array}\right.
$$

and the bounded strong quadratic growth condition means that

$$
\left\{\begin{array}{l}
\text { For any } N>0, \text { there exist } \alpha_{N}>0, \varepsilon_{N}>0: J(w) \geq J(\bar{w})+\frac{1}{2} \alpha_{N}\|w-\bar{w}\|_{2}^{2} \\
\text { for all } w \in F(P),\|y-\bar{y}\|_{\infty}<\varepsilon_{N},\|w\|_{\infty} \leq N .
\end{array}\right.
$$

We now recall the formulation of Pontryagin's principle at the point $\bar{w} \in F(P)$. The negative dual cone to $K$ (set of vectors of $\mathbb{R}^{r *}$ having a nonpositive duality product with each elements of $K)$ is $K^{-}=\mathbb{R}^{r_{1} *} \times \mathbb{R}_{+}^{r_{2} *}$; its elements are denoted $\mu=\left(\mu_{1}, \ldots, \mu_{r}\right)$. We say that $(z, \mu) \in K \times K^{-}$is a complementary pair if $\mu_{i} z_{i}=0$, for $i=1, \ldots, r$. The normal cone to $K$ at the point $z \in K$ is the set of elements of negative dual cone that are complementary to $z$. In particular, the expression of the normal cone to $K$ at $\Phi(\bar{\eta})$

$$
N_{K}(\Phi(\bar{\eta}))=\left\{\mu \in \mathbb{R}^{r *} ; \mu_{i} \geq 0, \mu_{i} \Phi_{i}(\bar{\eta})=0, i>r_{1}\right\} .
$$

Let the end-point Lagrangian be defined by (we set $\mu_{0}=1$ since we will restrict the analysis to qualified problems):

$$
\Phi^{\mu}\left(y_{0}, y_{T}\right):=\phi\left(y_{0}, y_{T}\right)+\sum_{i=1}^{r} \mu_{i} \Phi_{i}\left(y_{0}, y_{T}\right) .
$$

Consider the Hamiltonian function $H: \mathbb{R}^{m} \times \mathbb{R}^{n} \times \mathbb{R}^{n *}$ defined by

$$
H(u, y, p)=\ell(u, y)+p f(u, y) .
$$

Set $\mathcal{P}:=W^{1, \infty}\left(0, T ; \mathbb{R}^{n *}\right)$. For any $\mu \in \mathbb{R}^{r *}$ and $p \in \mathcal{P}$, consider the following set of relations: (recalling that $\bar{\eta}=(\bar{y}(0), \bar{y}(T))$ ):

(i) $-\dot{p}(t)=H_{y}\left(\bar{w}(t), p(t), \mu_{0}\right)$, a.a. $t \in(0, T)$;

(ii) $p(T)=\Phi_{y_{T}}^{\mu}(\bar{\eta})$;

(iii) $\quad p(0)=-\Phi_{y_{0}}^{\mu}(\bar{\eta})$. 
We call costate associated with $\mu$ at the point $\bar{w} \in F(P)$, and denote by $p^{\mu}$, the unique solution in $\mathcal{P}$ (since this reduces to the Cauchy problem for a linear o.d.e. with measurable and bounded coefficients) of the backward equation (2.13)(i-ii). Obviously, the mapping $\mu \rightarrow p^{\mu}$ is affine. We consider (2.13)(iii) as a necessary optimality condition.

Definition 2.1. Let $\bar{w} \in F(P)$. We say that $\mu \in N_{K}(\Phi(\bar{\eta}))$ is a (regular) Pontryagin multiplier associated with $\bar{w}$ if the associated costate $p^{\mu}$ satisfies (2.13)(iii), and is such that the following Hamiltonian inequality holds:

$$
H\left(\bar{u}(t), \bar{y}(t), p^{\mu}(t)\right) \leq H\left(v, \bar{y}(t), p^{\mu}(t)\right), \quad \text { for all } v \in U \text {, a.a. } t \in(0, T) .
$$

We denote by $M^{P}(\bar{w})$ the set of Pontryagin multipliers associated with $\bar{w}$; if this closed convex set is nonempty, we say that $\bar{w}$ satisfies Pontryagin's principle (in qualified form).

REMARK 2.2. If $\bar{w} \in F(P)$ and $\mu \in M^{P}(\bar{w})$, then we know that the function

$$
\bar{h}(\mu, t):=\inf _{v \in U} H\left(v, \bar{y}(t), p^{\mu}(t)\right), \quad t \in[0, T],
$$

is equal for all $t \in[0, T]$ to some constant $c_{\mu} \in \mathbb{R}$. By (2.13)(i-ii) $\mu \rightarrow c_{\mu}$ is affine. Set

$$
h(v, \mu, t):=H\left(v, \bar{y}(t), p^{\mu}(t)\right), \quad t \in(0, T) .
$$

By (2.14), we have that $h(\bar{u}(t), \mu, t)=\bar{h}(\mu, t)$ for a.a. $t \in(0, T)$. Define

$$
U_{N}:=U \cap B(0, N), \text { where } N>\|\bar{u}\|_{\infty} .
$$

Then there exists $\tilde{u}:[0, T] \rightarrow U_{N}$, a.e. equal to $\bar{u}$, such that

$$
h(\tilde{u}(t), \mu, t):=\bar{h}(\mu, t), \quad \text { for all } t \in[0, T] .
$$

It is known that Pontryagin's principle, in a non qualified form, is satisfied by Pontryagin solutions of $(P)$. The qualified form is satisfied under some qualification conditions to be seen later.

2.2. Hamiltonian functions with a unique minimum. If $A$ is a convex subset of a finite-dimensional space, we denote by $\operatorname{ri}(A)$ its relative interior, in the sense of convex analysis (the interior of $A$, in the topology induced by its affine hull). A relatively interior Pontryagin multiplier, i.e., some $\mu \in \operatorname{ri}\left(M^{P}(\bar{w})\right)$, obtains an increase of Hamiltonian of the same growth rate as the maximum over all Pontryagin multipliers:

Lemma 2.3. Assume that $\bar{w} \in F(P)$ satisfies Pontryagin's principle. Let $M^{C}(\bar{w})$ be a nonempty, convex and compact subset of $M^{P}(\bar{w})$, and $\bar{\mu} \in \operatorname{ri}\left(M^{C}(\bar{w})\right)$. Then there exists $\beta>0$ such that, for a.a. $t$, and any $v \in U$ :

$$
\begin{aligned}
& H\left(v, \bar{y}(t), p^{\bar{\mu}}(t)\right)-H\left(\bar{u}(t), \bar{y}(t), p^{\bar{\mu}}(t)\right) \\
& \quad \geq \max _{\mu \in M^{C}(\bar{w})}\left(H\left(v, \bar{y}(t), p^{\mu}(t)\right)-H\left(\bar{u}(t), \bar{y}(t), p^{\mu}(t)\right)\right),
\end{aligned}
$$

and also

$$
\beta \mu_{i} \leq \bar{\mu}_{i}, \quad \text { for all } i>r_{1} \text {. }
$$


The uniqueness of the minimum of the Hamiltonian function for all times $t$ implies that the control is continuous.

Given a point $\bar{w} \in F(P)$ such that the set $M^{P}(\bar{w})$ is nonempty and $N>$ $\|u\|_{\infty}$, we say that $\mu \in M^{P}(\bar{w})$ satisfies the hypothesis of unique minimum of the Hamiltonian over $U_{N}$ if the associated costate $p^{\mu}$ is such that, for all $t \in[0, T]$, the function $h(\cdot, \mu, t)=H\left(\cdot, \bar{y}(t), p^{\mu}(t)\right)$ has a unique minimum over $U_{M}$. Similarly, for $\mu \in M^{P}(\bar{w})$, we define the hypothesis of unique minimum of the Hamiltonian over the whole set $U$.

Remark 2.4. If for some $N>\|u\|_{\infty}$ and $\mu \in M^{P}(\bar{w})$ the hypothesis of unique minimum of the Hamiltonian over $U_{N}$ holds, then (i) by Lemma 2.3, it holds for any element of $\operatorname{ri}\left(M^{P}(\bar{w})\right)$, and (ii), for given $\varepsilon>0$ and $N>0$, there exists $\varepsilon_{N}>0$ such that

$$
\left\{\begin{array}{l}
H\left(v, \bar{y}(t), p^{\mu}(t)\right) \geq H\left(v, \bar{y}(t), p^{\mu}(t)\right)+\varepsilon_{N} \\
\text { whenever } v \in U_{N} ;|v-\bar{u}(t)| \geq \varepsilon, \text { for all } t \in[0, T] .
\end{array}\right.
$$

Lemma 2.5. Let the set $M^{P}(\bar{w})$ be nonempty at the point $\bar{w} \in F(P)$, and there exist $N>\|u\|_{\infty}$ and $\mu \in M^{P}(\bar{w})$ such that $\mu$ satisfies the hypothesis of unique minimum of the Hamiltonian over $U_{N}$. Then (one representative of) $\bar{u}(t)$ is a continuous function of time, equal to this unique minimum.

We define a bounded strong, Pontryagin, weak perturbation of $\bar{w} \in F(P)$ as a sequence $w_{k} \in F(P)$ associated with the corresponding optimality concept, i.e., bounded in $\mathcal{W}$ and such that $y_{k} \rightarrow \bar{y}$ uniformly, and additionally, $u_{k} \rightarrow u$ in $L^{1}$ (uniformly) in the case of Pontryagin (weak) perturbation.

LEMMA 2.6. Under the assumptions of the previous lemma, if $w_{k} \in F(P)$ is a bounded strong perturbation of $\bar{w}$ such that $\left\|u_{k}\right\|_{\infty}<N$ for all $k$, the following conditions are equivalent:

(i) $\lim \sup J\left(w_{k}\right) \leq J(\bar{w})$,

(ii) $\lim _{k}^{k} J\left(w_{k}\right)=J(\bar{w})$,

(iii) $\lim _{k} \int_{0}^{T}\left[H\left(u_{k}, \bar{y}, p^{\mu}\right)-H\left(\bar{u}, \bar{y}, p^{\mu}\right)\right] \mathrm{d} t=0(k \rightarrow \infty)$,

(iv) $u_{k} \rightarrow \bar{u}$ a.e.

Corollary 2.7. Let $\bar{w} \in F(P)$, satisfying Pontryagin's principle, be such that the hypothesis of unique minimum of the Hamiltonian over the whole set $U$ holds for some $\mu \in M^{P}(\bar{w})$. Then

(i) any bounded strong perturbation of $\bar{w}$ is a Pontryagin perturbation,

(ii) $\bar{w}$ is a bounded strong minimum iff it is a Pontryagin minimum.

Definition 2.8. We say that the Hamiltonian function satisfies a (local) quadratic growth condition for $\mu \in M^{P}(\bar{w})$ if there exist $\alpha>0$ and $\varepsilon>0$ such that

$$
\left\{\begin{array}{l}
H\left(v, \bar{y}(t), p^{\mu}(t)\right) \geq H\left(\bar{u}(t), \bar{y}(t), p^{\mu}(t)\right)+\alpha|v-\bar{u}(t)|^{2} \\
\text { whenever } v \in U ;|v-\bar{u}(t)| \leq \varepsilon, \text { for all } t \in[0, T]
\end{array}\right.
$$

REMARK 2.9. In view of Lemma 2.3, we have that, if $M^{C}(\bar{w})$ is a nonempty, convex and compact subset of $M^{P}(\bar{w})$, and there exist $\alpha>0$ and $\varepsilon>0$ such that

$$
\left\{\begin{array}{l}
\max _{\mu \in M^{C}(\bar{w})}\left(H\left(v, \bar{y}(t), p^{\mu}(t)\right)-H\left(\bar{u}(t), \bar{y}(t), p^{\mu}(t)\right)\right) \geq \alpha|v-\bar{u}(t)|^{2} ; \\
\quad \text { for all } v \in U,|v-\bar{u}(t)| \leq \varepsilon, t \in[0, T],
\end{array}\right.
$$


then the Hamiltonian function satisfies the quadratic growth condition, for any $\mu \in \operatorname{ri}\left(M^{P}(\bar{w})\right)$.

If, for all $t \in[0, T], h(v, \mu, t)$ has a unique minimum at $\bar{u}(t)$ over $U_{N}$ (with $N>$ $\left.\|\bar{u}\|_{\infty}\right)$, then by $(2.21)$, the quadratic growth condition for Hamiltonian functions implies

$$
\left\{\begin{array}{l}
H\left(v, \bar{y}(t), p^{\mu}(t)\right) \geq H\left(v, \bar{y}(t), p^{\mu}(t)\right)+\min \left(\alpha|v-\bar{u}(t)|^{2}, \varepsilon_{N}\right) \\
\text { whenever } v \in U_{N}, \text { for all } t \in[0, T]
\end{array}\right.
$$

\section{Inequality control constraints}

3.1. Robinson qualification condition. Lagrange and Pontryagin multipliers. We assume in this section that the control constraints are parameterized by finitely many inequalities:

$$
U:=\left\{u \in \mathbb{R}^{m} ; g(u) \leq 0\right\},
$$

where $g: \mathbb{R}^{m} \rightarrow \mathbb{R}^{q}$ is a $C^{2}$ mapping. In other words, the control constraints are defined by

$$
g_{i}(u(t)) \leq 0, \quad \text { for a.a. } t \in(0, T), \quad i=1, \ldots, q .
$$

We consider the "abstract" formulation where the state is a function of initial state and control. So we may write the state as $y_{u, y_{0}}(t)$, and define $G: \mathcal{U} \times \mathbb{R}^{n} \rightarrow \mathbb{R}^{r}$ by

$$
G_{i}\left(u, y_{0}\right):=\Phi_{i}\left(y_{0}, y_{u, y_{0}}(T)\right), \quad i=1, \ldots, r .
$$

By $G_{1: r_{1}}\left(u, y_{0}\right)$ we denote the (vertical) vector of components 1 to $r_{1}$ of $G\left(u, y_{0}\right)$. We say that the following Robinson qualification condition [17] (a natural infinite dimension generalization of the Mangasarian-Fromovitz condition [12]) holds at $\bar{w} \in F(P)$ if

$G_{1: r_{1}}^{\prime}\left(\bar{u}, \bar{y}_{0}\right)$ is onto, there exists $\beta>0$ and $\left(\bar{v}, \bar{z}_{0}\right) \in \operatorname{Ker} G_{1: r_{1}}^{\prime}\left(\bar{u}, \bar{y}_{0}\right)$; $g(\bar{u}(t))+g^{\prime}(\bar{u}(t)) \bar{v}(t) \leq-\beta, \quad$ for a.a. $t \in[0, T]$,

$$
G_{i}^{\prime}\left(\bar{u}, \bar{y}_{0}\right)\left(\bar{v}, \bar{z}_{0}\right) \quad \leq-\beta, \quad \text { for all } i>r_{1} \text { such that } G_{i}\left(\bar{u}, \bar{y}_{0}\right)=0 .
$$

In the sequel, we assume that at the feasible point $\bar{w}$ condition (3.4) holds.

Define the augmented Hamiltonian function by

$$
H^{a}(u, y, p, \lambda):=H(u, y, p)+\lambda g(u)=\ell(u, y)+p f(u, y)+\lambda g(u),
$$

where $u \in \mathbb{R}^{m}, y \in \mathbb{R}^{n}, p \in \mathbb{R}^{n *}$, and $\lambda \in \mathbb{R}^{q *}$. Given $\bar{w}=(\bar{u}, \bar{y}) \in F(P)$, we recall that the set of normal directions to $K$ at the point $\Phi(\bar{\eta})$ was defined in (2.10). The costate $p^{\mu} \in \mathcal{P}$ associated with $\mu \in N_{K}(\Phi(\bar{\eta}))$ was defined as the solution of (2.13). For $\bar{w} \in F(P)$ and $\mu \in N_{K}(\Phi(\bar{\eta}))$, define

$$
\begin{aligned}
\Lambda_{t}(\bar{w}, \mu) & :=\left\{\lambda \in \mathbb{R}_{+}^{q *} \cap g(\bar{u}(t))^{\perp} ; H_{u}^{a}\left(\bar{w}(t), p^{\mu}(t), \lambda\right)=0\right\}, \\
M^{L}(\bar{w}) & :=\left\{(\lambda, \mu) ; \mu \in N_{K}(\Phi(\bar{\eta})) ; \lambda \in L^{\infty}\left(0, T, \Lambda_{t}(\bar{w}, \mu)\right)\right\} .
\end{aligned}
$$

We call $M^{L}(\bar{w})$ the set of first-order (Lagrange) multipliers, and say that $(\bar{w}, p, \lambda, \mu)$ is a first-order extremal if $\bar{w} \in F(P),(\lambda, \mu) \in M^{L}(\bar{w})$, and $p=p^{\mu}$ is the associated costate.

TheOrem 3.1. Let $\bar{w}$ be a weak minimum of $(P)$. Then

(i) the set $M^{L}(\bar{w})$ is nonempty and bounded, and

(ii) $M^{P}(\bar{w})$ is a (possibly empty) subset of the projection of $M^{L}(\bar{w})$ under the mapping $(\lambda, \mu) \rightarrow \mu$. 
3.2. Restoration property. Relations between weak and bounded strong quadratic growth conditions. Given $\bar{w} \in F(P)$ such that $M^{P}(\bar{w}) \neq \emptyset$, and an arbitrary $\mu \in \operatorname{ri}\left(M^{P}(\bar{w})\right)$ (the relative interior of $M^{P}(\bar{w})$ ), denote the set of strictly (non strictly) complementary active constraints by

$$
\begin{aligned}
& I_{+}:=\left\{1, \ldots, r_{1}\right\} \cup\left\{r_{1}<i \leq r ; \mu_{i}>0\right\}, \\
& I_{0}:=\left\{r_{1}<i \leq r ; \Phi_{i}(\bar{\eta})=0\right\} \backslash I_{+} .
\end{aligned}
$$

In view of (2.20), all $\mu \in \operatorname{ri}\left(M^{P}(\bar{w})\right)$ have the same set of positive components, as can be easily checked, so that the definition does not depend on the choice of the particular $\mu$. Define

$$
K_{+}:=\left\{\theta \in \mathbb{R}^{r} ; \theta_{i}=0, i \in I_{+}, \theta_{i} \leq 0, i \in I_{0}\right\} .
$$

Note that, if $\mu \in \operatorname{ri}\left(M^{P}(\bar{w})\right)$, then $K_{+}=K \cap \mu^{\perp}$. The function

$$
d(\eta):=\sum_{i \in I_{0}} \Phi_{i}(\eta)_{+}+\sum_{i \in I_{+}}\left|\Phi_{i}(\eta)\right|
$$

is the distance of the initial-final state constraint to the set $K_{+}$, in the $L^{1}\left(\mathbb{R}^{r}\right)$ norm (the unique projection of $\theta \in \mathbb{R}^{r}$ in this norm being $\theta^{\prime}$ defined by $\theta_{i}^{\prime}=0$ if $i \in I_{+}$, and $\theta_{i}^{\prime}=\min \left(\theta_{i}, 0\right)$ otherwise). The following is called the Pontryagin norm:

$$
\|w\|_{P}:=\|u\|_{1}+\|y\|_{\infty} .
$$

Definition 3.2. We say that the restoration property (for the initial-final state constraints) is satisfied at $\bar{w} \in F(P)$ for $\bar{\mu} \in M^{P}(\bar{w})$ in the Pontryagin sense if there exists $\varepsilon>0$ and $\varepsilon_{B}>0$ such that, for any trajectory $w$ such that $\|w-\bar{w}\|_{P} \leq \varepsilon_{P}$ and $u(t) \in U$ a.e., and measurable set $B \subset(0, T)$ such that mes $(B) \leq \varepsilon_{B}$ over which $u$ and $\bar{u}$ coincide, there exists $w^{\prime} \in F(P)$ such that $u^{\prime}=\bar{u}$ on $B$ and

$$
\left\|w^{\prime}-w\right\|_{\infty}=O(d(\eta)) ; \quad J\left(w^{\prime}\right)=J^{\mu}(w)+O\left(\|w-\bar{w}\|_{P} d(\eta)\right) .
$$

Let us give a sufficient condition for the restoration property. For any $\varepsilon_{R}>0$, we denote the kernel of derivatives of almost active control constraints (relative to $\bar{w} \in \mathcal{W})$ by:

$$
\mathcal{U}_{\varepsilon_{R}}:=\left\{v \in \mathcal{U} ; g_{i}^{\prime}(\bar{u}(t)) v(t)=0 \text { whenever } g_{i}(\bar{u}(t)) \geq-\varepsilon_{R}, \text { for a.a. } t\right\} .
$$

Set $E_{\varepsilon_{R}}:=\mathcal{U}_{\varepsilon_{R}} \times \mathbb{R}^{n}, \bar{e}:=(\bar{u}, \bar{y}(0))$. The Mangasarian-Fromovitz qualification condition [12] at $\bar{w} \in F(P)$ (for constraints on initial-final state in $K_{+}$, over the Banach space $\left.E_{\varepsilon_{R}}\right)$ can be formulated as

$$
\left\{\begin{array}{l}
\text { There exists } \varepsilon_{R}>0, \text { such that over } E_{\varepsilon_{R}} \text { the following conditions } \\
\text { hold: } \\
\text { (i) There exists }\left\{e^{j}\right\} \subset E_{\varepsilon_{R}}, j=1, \ldots,\left|I_{+}\right| \text {, such that } \\
\quad\left\{G_{I_{+}}^{\prime}(\bar{e}) e^{j}\right\}_{j=1, \ldots,\left|I_{+}\right|} \text {is of rank }\left|I_{+}\right|, \\
\text {(ii) There exists } e^{0} \in E_{\varepsilon_{R}} \text { such that } G_{I_{+}}^{\prime}(\bar{e}) e^{0}=0 ; G_{I_{0}}^{\prime}(\bar{e}) e^{0}<0 .
\end{array}\right.
$$

We first note that this condition implies the uniqueness of the " $\mu$ part" of the multiplier.

Lemma 3.3. Let $\bar{w} \in F(P)$ satisfy (3.11). Let $\left(\lambda^{i}, \mu^{i}\right) \in M^{P}(\bar{w})$, for $i=1,2$. Then $\mu^{1}=\mu^{2}$.

The next lemma shows that (3.11) is a sufficient condition for the restoration property. 
Lemma 3.4. Let $\bar{w} \in F(P)$ satisfy (3.11). Then the restoration property (Definition 3.2) is satisfied in the Pontryagin sense.

As the following theorem shows, the quadratic growth condition for the Hamiltonian makes a bridge between the notions of weak and bounded strong quadratic growth.

TheOrem 3.5. Let $\bar{w} \in F(P)$ satisfy the qualification condition (3.4). Then:

a) The bounded strong quadratic growth condition at $\bar{w}$ implies the following three conditions:

(i) weak quadratic growth condition (2.8),

(i) the set $M^{P}(\bar{w})$ is nonempty, and for any $\mu$ in its relative interior, the Hamiltonian function satisfies the local quadratic growth condition (2.22), and

(iii) this multiplier $\mu$ satisfies the hypothesis of unique minimum of the Hamiltonian over the whole set $U$.

b) Conversely, if (i)-(iii) hold as well as the restoration property (3.9), then the bounded strong quadratic growth condition holds at $\bar{w}$.

\subsection{Second-order conditions.}

Critical directions. Since the qualification hypothesis (3.4) is a particular case of Robinson's qualification condition, the second-order necessary optimality condition due to Cominetti [3] (see also [2, Thm. 3.45]) holds. We denote $\bar{y}(0)$ as $\bar{y}_{0}$, and set $\hat{J}\left(u, y_{0}\right):=J\left(u, y_{u, y_{0}}\right)$, where $y_{u, y_{0}}$ is the solution of the state equation (2.2) with initial condition $y(0)=y_{0}$. Recall that $G$ was defined in (3.3). In order to state the mentioned conditions, let us define the weighted functions

$$
G^{\mu}\left(u, y_{0}\right):=\hat{J}\left(u, y_{0}\right)+\sum_{i=1}^{r} \mu_{i} G_{i}\left(u, y_{0}\right)
$$

the Lagrangian function of the problem $L: \mathcal{U} \times \mathbb{R}^{n} \times L^{\infty}\left(0, T, \mathbb{R}^{q *}\right) \times \mathbb{R}^{r *}$ defined by

$$
L\left(u, y_{0}, \lambda, \mu\right):=G^{\mu}\left(u, y_{0}\right)+\int_{0}^{T} \lambda(t) g(u(t)) \mathrm{d} t,
$$

the set of active inequalities at time $t$ and for the initial-final state constraints:

$$
I_{t}:=\left\{1 \leq i \leq q ; g_{i}(\bar{u}(t))=0\right\} ; \quad I_{F}:=\left\{r_{1}+1 \leq j \leq r ; G_{j}\left(\bar{u}, \bar{y}_{0}\right)=0\right\} .
$$

Set $\mathcal{U}_{2}:=L^{2}\left(0, T, \mathbb{R}^{m}\right)$. The linear mappings $\hat{J}^{\prime}\left(\bar{u}, \bar{y}_{0}\right)$ and $G^{\prime}\left(\bar{u}, \bar{y}_{0}\right)$ have a unique extension over $\mathcal{U}_{2} \times \mathbb{R}^{n}$ that will be denoted in the same way. We define the set of extended tangent directions to the control and initial-final state constraints (they are extended in the sense that we take $L^{2}$ spaces instead of $L^{\infty}$ ):

$$
T_{g}(\bar{u}):=\left\{v \in \mathcal{U}_{2} ; g_{I_{t}}^{\prime}(\bar{u}(t)) v(t) \leq 0 \text {, a.a. } t \in(0, T)\right\},
$$

$$
T_{\Phi}\left(\bar{u}, \bar{y}_{0}\right):=\left\{\left(v, z_{0}\right) \in \mathcal{U}_{2} \times \mathbb{R}^{n} ; G_{1: r_{1}}^{\prime}\left(\bar{u}, \bar{y}_{0}\right)\left(v, z_{0}\right)=0 ; G_{I_{F}}^{\prime}\left(\bar{u}, \bar{y}_{0}\right)\left(v, z_{0}\right) \leq 0\right\},
$$

the set of extended critical directions:

$$
C_{2}\left(\bar{u}, \bar{y}_{0}\right):=\left\{\left(v, z_{0}\right) \in T_{\Phi}\left(\bar{u}, \bar{y}_{0}\right) ; v \in T_{g}(\bar{u}) ; \hat{J}^{\prime}\left(\bar{u}, \bar{y}_{0}\right)\left(v, z_{0}\right) \leq 0\right\} .
$$

The set of critical directions (in the original space) is

$$
C_{\infty}\left(\bar{u}, \bar{y}_{0}\right):=\left\{\left(v, z_{0}\right) \in C_{2}\left(\bar{u}, \bar{y}_{0}\right) ; \quad v \in L^{\infty}\left(0, T, \mathbb{R}^{m}\right)\right\} .
$$


Finally $T_{-}^{2}\left(g(\bar{u}), g^{\prime}(\bar{u}) v\right)$ stands for the second-order tangent set to $L^{\infty}\left(0, T, \mathbb{R}_{-}^{q}\right)$ at the point $g(\bar{u})$, in the direction $g^{\prime}(\bar{u}) v$, i.e., for $s>0$ :

$$
T_{-}^{2}\left(g(\bar{u}), g^{\prime}(\bar{u}) v\right)=\left\{w \in L^{\infty}\left(0, T, \mathbb{R}^{q}\right) ; \underset{t}{\operatorname{supess}}\left(g(\bar{u})+s g^{\prime}(\bar{u}) v+\frac{1}{2} s^{2} w\right) \leq o\left(s^{2}\right)\right\} .
$$

By Cominetti [3], we have that (as usual, $\sigma_{K}(\cdot)$ denotes the support function to a set $K$, i.e., the supremum of duality products with elements of $K$ and the corresponding term in (3.20) is called "sigma-term"):

THEOREM 3.6. Let $\bar{w}=(\bar{u}, \bar{y})$ be a weak solution of $(P)$. Then the set $M^{L}(\bar{w})$ is nonempty and

$$
\begin{array}{r}
\max _{(\lambda, \mu) \in M^{L}(\bar{w})}\left(L_{\left(u, y_{0}\right)^{2}}\left(\bar{u}, \bar{y}_{0}, \lambda, \mu\right)\left(v, z_{0}\right)^{2}-\sigma\left(\lambda, T_{-}^{2}\left(g(\bar{u}), g^{\prime}(\bar{u}) v\right)\right) \geq 0\right. \\
\text { for all }\left(v, z_{0}\right) \in C_{\infty}\left(\bar{u}, \bar{y}_{0}\right) .
\end{array}
$$

Note that the second-order tangent set in $L^{\infty}$ has no practical characterization (see however [4]). The proof of Theorem 3.6 is a standard application of [3] (note that since the constraint on the initial-final state consist in a finite number of inequalities they have no contribution to the "sigma term"). We next formulate a stronger result. Since the sigma term is nonpositive, and $C_{\infty}\left(\bar{u}, \bar{y}_{0}\right) \subset C_{2}\left(\bar{u}, \bar{y}_{0}\right)$, a sufficient condition for $(3.20)$ is

$\Omega\left(v, z_{0}\right):=\max _{(\lambda, \mu) \in M^{L}(\bar{w})} L_{\left(u, y_{0}\right)^{2}}\left(\bar{u}, \bar{y}_{0}, \lambda, \mu\right)\left(v, z_{0}\right)^{2} \geq 0, \quad$ for all $\left(v, z_{0}\right) \in C_{2}\left(\bar{u}, \bar{y}_{0}\right)$.

Note that the above condition makes sense since, for any $(\lambda, \mu) \in M^{L}(\bar{w})$, the quadratic form $\left(v, z_{0}\right) \mapsto L_{\left(u, y_{0}\right)^{2}}\left(\bar{u}, \bar{y}_{0}, \lambda, \mu\right)\left(v, z_{0}\right)^{2}$, defined over $\mathcal{U} \times \mathbb{R}^{n}$, has a unique extension over $\mathcal{U}_{2} \times \mathbb{R}^{n}$.

THEOREM 3.7. Let $\bar{w}=(\bar{u}, \bar{y})$ be a weak solution of $(P)$, satisfying the strong qualification hypothesis (3.11). Then $M^{L}(\bar{w}) \neq \emptyset$ and condition (3.21) holds.

REMARK 3.8. The method of proof is a variant of the one used for "extended polyedricity", see [2, Sec. 3.2.3]. The basic concept there is the one of radial critical directions, i.e., critical directions $v$ for which there exists $\beta>0$ such that (in our notations) $g(u)+\beta g^{\prime}(u) v \leq 0$. Here the $L^{\infty}$ smoothness of the multiplier compensates the lack of density in $L^{\infty}$.

Finally, we formulate the characterization of the weak quadratic growth condition at the point $\bar{w} \in F(P)$, using the following condition:

$$
\text { For some } \alpha>0, \Omega\left(v, z_{0}\right) \geq \alpha\left(\|v\|_{2}+\left|z_{0}\right|\right)^{2}, \quad \text { for all }\left(v, z_{0}\right) \in C_{2}\left(\bar{u}, \bar{y}_{0}\right) \text {. }
$$

THEOREM 3.9. Let $\bar{w} \in F(P)$, with associated non empty set of Lagrange multipliers, satisfy the strong qualification hypothesis (3.11), with partition $\left(I_{+}, I_{0}\right)$ now defined by the fact that $i \in I_{0}$ iff each Lagrange multiplier $(\lambda, \mu)$ is such that $\mu_{i}=0$ for $i>r_{1}$. Then $\bar{w}$ satisfies the weak quadratic growth condition iff the following two conditions hold:

(i) local quadratic growth (2.22) of Hamiltonian function along the trajectory,

(ii) uniform quadratic growth (3.22) along critical directions.

The proofs of these results will be published in $[\mathbf{1}]$. 


\section{References}

[1] J.F. Bonnans and N.P. Osmolovskii, Second-order analysis of optimal control problems with control and initial-final state constraints. INRIA Report RR 6707, October 2008.

[2] J.F. Bonnans and A. Shapiro, Perturbation Analysis of Optimization Problems, SpringerVerlag, New York, 2000.

[3] R. Cominetti, Metric regularity and second order optimality conditions, J. Applied Math. Optimization 21 (1990), 265-287.

[4] R. Cominetti and J.P. Penot, Tangent sets to unilateral convex sets, Comptes Rendus de l'Académie des Sciences de Paris, Série I 321 (1995), 1631-1636.

[5] A.Ya. Dubovitski and A.A. Milyutin, Extremum problems with constraints, [in Russian], J. Comp. Sc. and Math. Phys. 5, (1965), no. 3, 395-453.

[6] E.M. Galeev, M.I Zelikin, S.V. Konyagin, G.G. Magaril-Ilyaev, N.P. Osmolovskii, V.Yu. Protasov, V.M. Tikhomirov, A.V. Fursikov, Optimal Control [in Russian], eds. N.P. Osmolovskii and V.M. Tikhomirov, MCCME, Moscow, 2008.

[7] M.R. Hestenes, Calculus of Variations and Optimal Control Theory, Wiley, New York, 1966.

[8] A. J. Hoffman, On approximate solutions of systems of linear inequalities, J. Res. National Bur. Standards No. 49 (1952), 263-265.

[9] A.D. Ioffe, Necessary and sufficient conditions for a local minimum I: A reduction theorem and first order conditions, II: Conditions of Levitin-Miljutin-Osmolovskii type, III: Second order conditions and augmented duality, SIAM Journal on Control Optimization 17 (1979), 245-250, 251-265 and 266-288.

[10] A.D. Ioffe, V.M. Tikhomirov, Theory of Extremal Problems, Nauka, Moscow, 1974; English transl., North-Holland, Amsterdam, 1979.

[11] E.S. Levitin, A.A. Milyutin, N.P. Osmolovskii, Conditions of high order for a local minimum in problem with constraints, Russian Math. Surveys 33 (1978), no. 6, 97-168.

[12] O. Mangasarian and S. Fromovitz, The Fritz-John necessary optimality conditions in the presence of equality and inequality constraints, J. Math. Analysis Applications 7 (1967), $37-47$.

[13] A.A. Milyutin, Maximum Principle in the General Optimal Control Problem [in Russian], Fizmatlit, Moscow, 2001.

[14] A.A. Milyutin and N.P. Osmolovskii, Calculus of Variations and Optimal Control, Translations of Math. Monographs, Vol. 180, American Math. Soc., Providence, 1998.

[15] N.P. Osmolovskii, On a system of linear inequalities on a convex set [in Russian], Usp. Mat. Nauk 32 (1977), no. 2, 223-224.

[16] N.P. Osmolovskii, Theory of higher order conditions in optimal control, Doctoral Thesis, MISI (Moscow Civil Engineering Institute), Moscow, 1988.

[17] S.M. Robinson, First order conditions for general nonlinear optimization, SIAM J. Applied Math 30 (1976), 597-607.

inRiA-Saclay and Centre de Mathématiques Appliquées, Ecole Polytechnique, route de Saclay, 91128 Palaiseau, France

E-mail address: Frederic.Bonnans@inria.fr

Systems Research Institute, ul. Newelska 6, 01-447, Warszawa, Poland; Polytechnika Radomska, 26-600 Radom, ul. Malczewskiego 20A, Poland; Akademia Podlaska, ul. 3 Maja 54, 08-110 Siedlce, Poland

E-mail address: osmolovski@ap.siedlce.pl

This is a free offprint provided to the author by the publisher. Copyright restrictions may apply. 